\title{
Successful Retrograde Dilation and Oesophageal Conservation after Failed Antegrade Management of a Reflux Stricture
}

In modern oesophageal practice, failure to dilate a reflux stricture by antegrade techniques is a very rare event (1). When a stricture is not dilatable, oesophagectomy and reconstruction with a visceral substitute is generally recommended $(2-5)$.

We report here the case of a 77 -year-old woman who was referred with a "tight oesophageal stricture, $25 \mathrm{~cm}$ from the dental margin". Prior to this, the referring surgeon had been unable to pass a dilator or a guide wire. A barium swallow series confirmed the tight stricture (Figure 1). Under general anaesthesia, the patient underwent flexible oesophagoscopy, which revealed a pinholesized high oesophageal stenosis. Dilation was attempted by two experienced consultant endoscopists, but was unsuccessful. A small open gastrotomy was made, and under direct vision, using a sterile flexible gastroscope, a guide wire was passed in a retrograde fashion through the stenosis. Using image intensification, successive metal olive dilators (Eder-Puestow) were passed over this guide wire to dilate the oesophagus to $26 \mathrm{Fr}$. A 16-gauge nasogastric tube was then passed retrogradely through the stenosis into the oropharynx. An 8-gauge nasogastric tube was passed through a cervical pharyngostomy and via the 16-gauge nasogastric tube into the stomach. A tube gastrostomy was carried out using a Depezzar-type catheter. A feeding tube jejunostomy was advanced via the gastrostomy through the duodenum (Figure 2).

She was initially fed enterally while recovering from these procedures. Nineteen days later, it was possible to dilate the stricture antegradely over a guide wire after removal of the pharyngostomy tube. She was allowed, and tolerated, a light oral diet the following day, and was transferred a day later to a nursing home for supervised recuperation. After three further dilations at monthly intervals, she was able to swallow a normal diet. This approach allowed successful management, conserving the oesophagus, of a peptic oesophageal stricture in a frail elderly patient, when the stricture had not been manageable using antegrade methods.

G. C. O'Sullivan, M. G. O'Brien

Dept. of Surgery, Mercy Hospital and National University of Ireland, Cork, Ireland

\section{References}

1. Hennessy TPJ, Cuschieri A. Benign oesophageal strictures. In: Hennessy TPJ, Cuschieri A, editors. Surgery of the oesophagus. London: Butterworth-Heinemann, 1992: 255-74.

2. Spechler SJ. Epidemiology and natural history of gastrooesophageal reflux disease. Digestion 1992; 51 (Suppl 1):24-9.

3. Morton LS, Fromkes JJ. Gastroesophageal reflux disease: diagnosis and medical therapy. Geriatrics 1993; 48: 60-6.

4. Csendes A, Braghetto I. Surgical management of esophageal strictures. Hepato-Gastroenterology 1992; 39: 502-10.

5. Bonavina L, Fontebasso V, Bardini R, et al. Surgical treatment of reflux stricture of the oesophagus. Br J Surg 1993; 80: 317-20.

Corresponding Author

G. C. O'Sullivan, M.Ch., Dept. of Surgery,

Mercy Hospital, Cork, Ireland

Fax: +353-21-276341

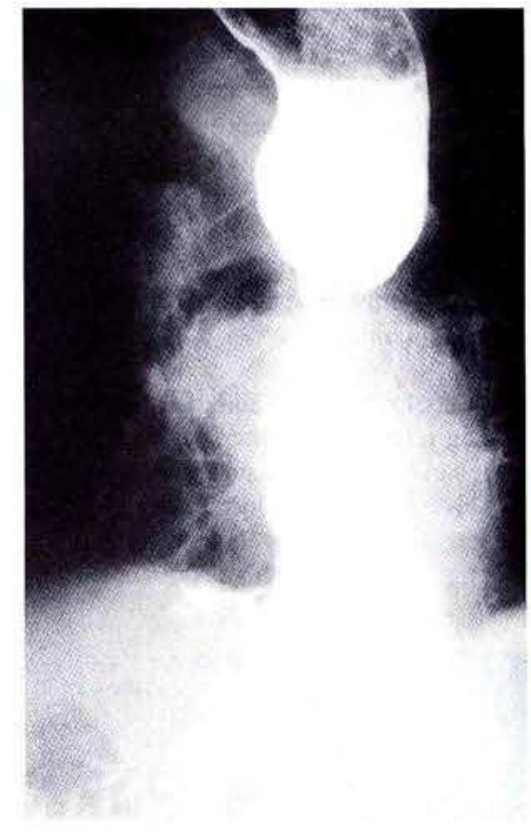

Figure 1: Contrast radiograph, showing a tight stricture above a fixed hiatus hernia.

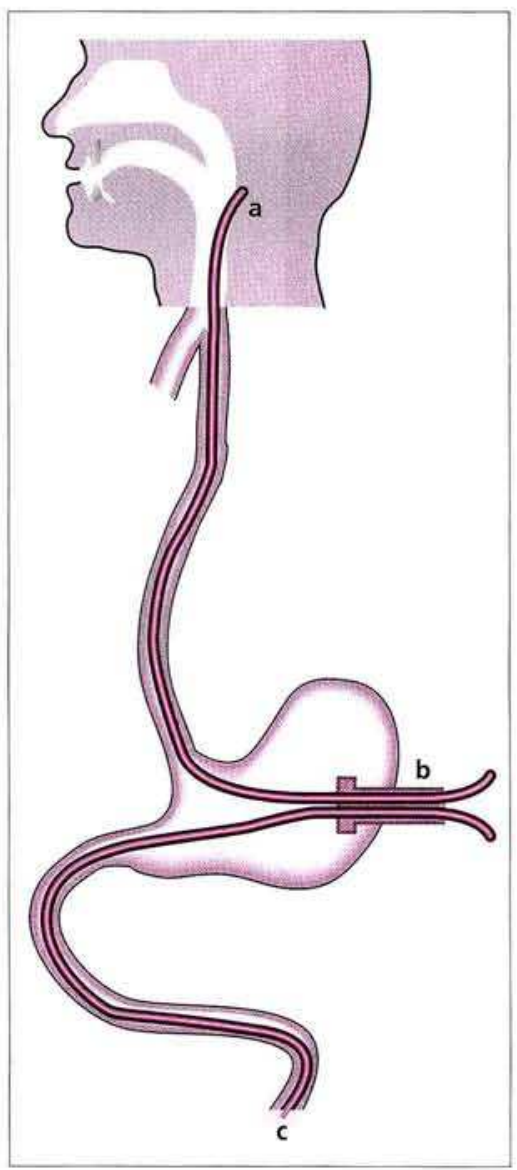

Figure 2: Diagram of the placement of the catheters after surgery; a pharyngostomy tube, b gastrostomy tube, c feeding jejunostomy tube. 